\title{
Signaling of the p21-activated kinase (PAK1) coordinates insulin-stimulated actin remodeling and glucose uptake in skeletal muscle cells
}

\author{
Ragadeepthi Tundugurua ${ }^{a}$, Tim T. Chiu ${ }^{b}$, Latha Ramalingam ${ }^{a, c}$, Jeffrey S. Elmendorf ${ }^{a, d}$, \\ Amira Klip $^{\mathrm{b}}$, and Debbie C. Thurmond ${ }^{\mathrm{a}, \mathrm{c}, \mathrm{d},{ }^{*}}$ \\ aDepartment of Biochemistry and Molecular Biology, Indiana University School of Medicine, \\ Indianapolis, IN, USA \\ ${ }^{b}$ Cell Biology Program, The Hospital for Sick Children, Toronto, ON, Canada \\ 'Department of Pediatrics, Indiana University School of Medicine, Indianapolis, IN, USA \\ dDepartment of Cellular and Integrative Physiology, Indiana University School of Medicine, \\ Indianapolis, IN, USA
}

\begin{abstract}
Skeletal muscle accounts for $\sim 80 \%$ of postprandial glucose clearance, and skeletal muscle glucose clearance is crucial for maintaining insulin sensitivity and euglycemia. Insulin-stimulated glucose clearance/uptake entails recruitment of glucose transporter 4 (GLUT4) to the plasma membrane (PM) in a process that requires cortical F-actin remodeling; this process is dysregulated in Type 2 Diabetes. Recent studies have implicated PAK1 as a required element in GLUT4 recruitment in mouse skeletal muscle in vivo, although its underlying mechanism of action and requirement in glucose uptake remains undetermined. Toward this, we have employed the PAK1 inhibitor, IPA3, in studies using L6-GLUT4-myc muscle cells. IPA3 fully ablated insulin-stimulated GLUT4 translocation to the PM, corroborating the observation of ablated insulin-stimulated GLUT4 accumulation in the PM of skeletal muscle from PAK $1^{-/}$knockout mice. IPA3-treatment also abolished insulin-stimulated glucose uptake into skeletal myotubes. Mechanistically, live-cell imaging of myoblasts expressing the F-actin biosensor LifeAct-GFP treated with IPA3 showed blunting of the normal insulin-induced cortical actin remodeling. This blunting was underpinned by a loss of normal insulin-stimulated cofilin dephosphorylation in IPA3-treated myoblasts. These findings expand upon the existing model of actin remodeling in glucose uptake, by placing insulin-stimulated PAK1 signaling as a required upstream step to facilitate actin remodeling and subsequent cofilin dephosphorylation. Active, dephosphorylated cofilin then provides the G-actin substrate for continued F-actin remodeling to facilitate GLUT4 vesicle translocation for glucose uptake into the skeletal muscle cell.
\end{abstract}

(C) 2014 Elsevier Inc. All rights reserved.

*Corresponding author. dthurmon@iu.edu (D.C. Thurmond). 


\section{Keywords}

F-actin remodeling; Diabetes; PAK1; L6-GLUT4myc muscle cells; Skeletal muscle; GLUT4 vesicle exocytosis; Small Rho family GTPase; Rac1

\section{Introduction}

Insulin maintains the glucose homeostasis of the body by mobilizing insulin-responsive glucose transporter 4 (GLUT4)-containing vesicles from intracellular compartments to the plasma membrane (PM) of muscle and adipose cells, facilitating glucose uptake into these tissues [1-3]. Defects and/or deficiencies in insulin-stimulated glucose uptake by these peripheral tissues promote the development of peripheral insulin resistance and pre-diabetes [4,5]. Skeletal muscle glucose uptake accounts for $\sim 80 \%$ of all insulin-stimulated uptake [6]. Under normal insulin-sensitive conditions, circulating insulin binds to the insulin receptor present on the extracellular surface of the skeletal muscle cell to initiate an intracellular signaling cascade which bifurcates downstream of phosphatidylinositol 3-kinase (PI3K) into at least two parallel pathways that lead to GLUT4 vesicle translocation, facilitating glucose uptake into the muscle cell [7,8]. Though the first pathway involving PI3K-Akt-AS160-Rab GTPase leading to GLUT4 vesicle translocation is well-studied, the second insulin-signaling arm involving PI3K-Rac1 and actin remodeling to GLUT4 vesicle mobilization in skeletal muscle remains incompletely characterized.

Rac1 is a small Rho family GTPase which is typically involved in the regulation of cytoskeletal reorganization and vesicular traffic in most cell types [9]. Growing evidence supports the involvement of Rac1 in insulin-induced GLUT4 vesicle translocation to the cell surface, both in cultured L6 muscle cells and mature primary skeletal muscle [10-12]. Knockdown or inhibition of Rac1 activity ablates GLUT4 translocation and subsequent glucose uptake [13]. Rac1 mediates this process by inducing cortical F-actin remodeling, which involves the recruitment of actin regulatory proteins such as cofilin and Arp2/3 to the actin filaments in skeletal muscle cells [14]. Additionally, Rac1 signals to p21-activated kinase 1 (PAK1) in skeletal muscle and facilitates its phosphorylation in response to insulin $[13,15]$. Insulin-stimulated PAK1 activation was decreased in human skeletal muscle of both acute (intralipid infusion) and chronic (obesity and Type 2 diabetes) insulin resistant states [13], implicating PAK1 as a required element in maintaining euglycemia and insulin sensitivity. However, the connections between PAK1, Rac1 and cortical F-actin remodeling to mediate insulin-dependent GLUT4 movement to the cell surface have yet to be established.

Several studies in mice depleted of PAK1 (whole-body PAK1 ${ }^{-/-}$knockout) support the requirement for PAK1 in the maintenance of glucose homeostasis in vivo, linked to its actions in islets [16,17] and skeletal muscle [16]. Although PAK1 function in islets appears to be linked to Cdc42 [18,19], its role in skeletal muscle is predicted to be linked instead to Rac1, given that Rac1 but not $\mathrm{Cdc} 42$ has been shown to participate in glucose uptake $[10,11]$. However, given the reported requirements for PAK1 in heart tissue, two cell types of the islet ( $\alpha$ - and $\beta$-cells) and intestinal cells [17,20-22], the metabolic derangement of the PAK1 KO mouse and the role of PAK1 in skeletal muscle has remained unresolved. In 
addition, PAK1 serves two roles, one as a kinase in signaling actions, and another as a scaffolding protein [23]. Moreover, it is unknown whether PAK1's signaling or scaffolding functions, or both, are required for GLUT4 vesicle translocation and glucose uptake in skeletal muscle for the regulation of whole-body glucose levels.

In the current study we tested the hypothesis that PAK1 signaling is required for insulinstimulated GLUT4 vesicle translocation to the cell surface by contributing to cortical actin remodeling in skeletal muscle cells. Towards this end, we employed an inhibitor of PAK signaling, IPA3, to distinguish PAK1's requirement for signaling from that of scaffolding. IPA3 is a selective inhibitor of the group I PAK isoforms; another PAK inhibitor, PF-3758309, acts on both group I and II PAKs. Skeletal muscle and L6-GLUT4myc clonal muscle cells were used, as both showed identical expression of the Group 1 PAK isoforms, of which only the PAK1 isoform was found to be of significance to insulin-stimulated GLUT4 vesicle translocation. Inhibition of PAK1 signaling function completely ablated insulin-dependent GLUT4 translocation and glucose uptake in L6-GLUT4myc muscle cells. Mechanistically, IPA3 blunted the insulin-induced dynamic rearrangement of cortical Factin in L6-GLUT4myc myoblasts. We further demonstrate that PAK1 signals to dephosphorylate cofilin, in a manner independent of LIM kinase, revealing an unusual signaling axis for actin remodeling in skeletal muscle cells.

\section{Materials and methods}

\subsection{Materials}

Rat L6 GLUT4-myc skeletal muscle cells expressing c-myc tagged GLUT4 protein were developed as described [24]. MEMa medium was purchased from Invitrogen (Carlsbad, CA). Porcine insulin, rabbit anti-actin antibody and 2-deoxyglucose were purchased from Sigma-Aldrich (St. Louis, MO). The LifeAct-GFP plasmid was kindly provided by Dr. Louis Philipson (University of Chicago, IL [25]), and the GFP-hPID and GFP-hPID-L107F plasmids were a gift from Dr. Jonathan Chernoff (Fox Chase Cancer Center, PA [26]). An inhibitor of the activation of group I PAKs (IPA3), phospho-PAK1 ${ }^{\mathrm{T} 423}$ and mouse anti-pcofilin antibody were purchased from Santa Cruz Biotech (Santa Cruz, CA). PhosphoPAK1 ${ }^{\mathrm{T} 423 / \text { phospho-PAK2 }}{ }^{\mathrm{T} 402}$, PAK1, PAK2, PAK3, cofilin, phospho-ERK1/2 $202 / \mathrm{Y} 204$ and ERK1/2 antibodies were obtained from Cell Signaling (Danvers, MA). Fetal bovine serum and goat anti-mouse horseradish peroxidase secondary antibody were obtained from Thermo-Fisher Scientific (Rockford, IL). Goat anti-rabbit horseradish peroxidase secondary antibody was purchased from Bio-Rad (Hercules, CA). Enhanced chemiluminescence reagent (ECL), ECL prime and SuperSignal ${ }^{\mathrm{TM}}$ Femto were purchased from GE Healthcare (Piscataway, NJ) and Pierce (Rockford, IL), respectively.

\subsection{Cell culture}

L6-GLUT4myc myoblasts were grown as monolayers in MEM-a medium supplemented with $10 \%$ fetal bovine serum and $1 \%(\mathrm{v} / \mathrm{v})$ antibiotic-antimycotic solution. L6-GLUT4-myc myoblasts at $40 \%$ confluency were differentiated into myotubes by incubation in MEM-a medium containing $2 \%$ fetal bovine serum $[27,28]$. For all the studies involving IPA3, L6GLUT4-myc myoblasts ( $90 \%$ confluency) were pre-incubated in serum-free medium for 3 
$\mathrm{h}$ with IPA3 added for the times indicated in the figures just prior to insulin stimulation (100 $\mathrm{nM}$ ). Cells were harvested in 1\% NP-40 lysis buffer containing 25 mM HEPES, $\mathrm{pH} 7.4,1 \%$ Nonidet P-40, $10 \%$ glycerol, $50 \mathrm{mM}$ sodium fluoride, $10 \mathrm{mM}$ sodium pyrophosphate, 137 $\mathrm{mM} \mathrm{NaCl}, 1 \mathrm{mM}$ sodium vanadate, $1 \mathrm{mM}$ phenylmethylsulfonyl fluoride, $10 \mu \mathrm{g} / \mathrm{ml}$ aprotinin, $1 \mu \mathrm{g} / \mathrm{ml}$ pepstatin, $5 \mu \mathrm{g} / \mathrm{ml}$ leupeptin and cleared of insoluble material by centrifugation at $13,000 \times g$ for $10 \mathrm{~min}$ at $4{ }^{\circ} \mathrm{C}$. Supernatant was used for immunoblot analyses. Cells were transfected with plasmid DNA using Effectene transfection reagent (Qiagen, Valencia, CA), Lipofectamine 2000 (LifeTechnologies, Grand Island, NY) or with siRNA oligonucleotides using Jet Prime transfection reagent according to the manufacturer's protocol (Polyplus transfection, NY, USA) as recently described [29]. siRNA oligonucleotide sequences used: siPAK2 sense $5^{\prime}$-ggucugucaucgacccuautt-3' and antisense $5^{\prime}$-auagggucgaugacagacctt- $3^{\prime}$; siControl sense $5^{\prime}$-uaaggcuaugaagagauactt- $3^{\prime}$ and antisense $5^{\prime}$-guaucucuucauagccuuatt- $3^{\prime}$, obtained from Qiagen.

\subsection{RNA isolation and qRT-PCR}

RNA was isolated from islets using the RNA easy Fibrous Tissue Minikit (Qiagen, Valencia, CA) and reverse-transcribed to cDNA using the Superscript First strand synthesis system (Invitrogen, Carlsbad, CA). PCR was performed using Biomix red for 30 cycles: 94 ${ }^{\circ} \mathrm{C}$ for $1 \mathrm{~min}, 56{ }^{\circ} \mathrm{C}$ for $1 \mathrm{~min}$, and $71{ }^{\circ} \mathrm{C}$ for $1 \mathrm{~min}$, with a final 10-min elongation at $71{ }^{\circ} \mathrm{C}$ and PCR products were visualized on $2 \%$ agarose gel. Primers used for the detection of PAK1 (forward: 5-tgtctgagaccccagcagta andreverse:5'-cccgagttggagtaacagga), PAK2(forward 5-aacaccagcactgaacacca and reverse $5{ }^{\prime}$-cttggcaccactgtcaacat), PAK3 (forward 5-gcagcacatcagtcgaatacca and reverse $5^{\prime}$-tttatttggtgcagctggt) and GAPDH (5'atggtgaaggtcggtgtgaacg and reverse $5^{\prime}$-gttgtcatggatgaccttggcc) were obtained from IDT (Coralville, IA). The qRT-PCR reaction was performed using CFX Connect Real-Time system (Bio-Rad, Hercules, CA) and amplifications were done using the Platinum SYBR Green qPCR SuperMix-UDG (Invitrogen, Carlsbad, CA). The thermal cycling conditions for the reaction were as follows: $50{ }^{\circ} \mathrm{C}$ for 2 -min hold (UDG incubation), $95{ }^{\circ} \mathrm{C}$ for 2-min hold, 40 cycles of $95^{\circ} \mathrm{C}$ for $15 \mathrm{~s}$, and $60^{\circ} \mathrm{C}$ for $30 \mathrm{~s}$. PCR products were visualized on $2 \%$ agarose gels. Relative quantification in gene expression levels were quantified using the $2^{-\Delta \mathrm{Ct}}$ method where relative mRNA levels of PAK1, 2 and 3 reported are normalized to GAPDH.

\subsection{Live-cell imaging}

L6-GLUT4myc myoblasts were seeded on MatTek glass bottom culture dishes at a density of 300,000 cells per $35 \mathrm{~mm}$ dish. At $\sim 40 \%$ confluency cells were transfected with LifeActGFP plasmid using Effectene transfection reagent (Qiagen, Valencia, CA). Live-cell imaging was performed on cells $48 \mathrm{~h}$ post-transfection. Briefly, on the day of the experiment the cells were pre-incubated in serum-free KRPH buffer $(120 \mathrm{mM} \mathrm{NaCl}, 2.5 \mathrm{mM} \mathrm{KCl}, 20$ mM HEPES, $1.2 \mathrm{mM} \mathrm{MgSO}_{4}, 1 \mathrm{mM} \mathrm{NaH}_{2} \mathrm{PO}_{4}$, and $2 \mathrm{mM} \mathrm{CaCl}_{2}$ ) supplemented with 5 $\mathrm{mM}$ D-glucose for $3 \mathrm{~h}$, then IPA3 or vehicle (DMSO) added for $50 \mathrm{~min}$. LifeAct-GFP imaging was performed on a custom spinning-disk confocal microscope with a heated $60 \times$ Plan Apo Lambda 1.4 NA objective lens and sample chamber with temperature, humidity and $\mathrm{CO}_{2}$ regulation built around a CSU-10 spinning disk confocal head (Yokogawa) which is controlled by NIS Elements AR v 4.10 (Nikon Instruments). Images were captured every 
$60 \mathrm{~s}$ starting $1 \mathrm{~min}$ before the addition of insulin and continued through until $10 \mathrm{~min}$ after the addition of insulin. Movies of each condition are shown as Supplemental data movies 14.

\subsection{Cell surface GLUT4myc detection}

Cell surface GLUT4myc detection was performed as described earlier [30]. Briefly, L6GLUT4-myc myoblasts or myotubes were pre-incubated in serum-free medium containing IPA3 $(25 \mu \mathrm{M})$ or vehicle (DMSO) for $40 \mathrm{~min}$ followed by insulin stimulation (100 $\mathrm{nM}$ for $20 \mathrm{~min}$ ), all at $37{ }^{\circ} \mathrm{C}$. Cells were then fixed with $4 \%$ paraformaldehyde in PBS for $20 \mathrm{~min}$ at room temperature (RT), blocked in Odyssey Blocking Buffer (LI-COR Biosciences, Lincoln, NE) for $1 \mathrm{~h}$ at RT and incubated with mouse anti-Myc antibody overnight at $4{ }^{\circ} \mathrm{C}$. Cells were extensively washed with PBS and then incubated with infrared (IR)-conjugated secondary antibody for $1 \mathrm{~h}$ at RT. Immunofluorescence intensity of the IR-conjugated secondary antibody was quantified using the LiCor infrared imaging system (LI-COR Biosciences, Lincoln, NE) and data normalized to SYTO 60 (Invitrogen, Carlsbad, CA), a red fluorescent nucleic acid stain.

\subsection{2-Deoxyglucose uptake assay}

The 2-deoxyglucose uptake assay was performed as described [31]. Briefly, L6 myotubes were pre-incubated in serum-free FCB buffer $\left(125 \mathrm{mM} \mathrm{NaCl}, 5 \mathrm{mM} \mathrm{KCl}, 1.8 \mathrm{mM} \mathrm{CaCl}_{2}\right.$, $2.6 \mathrm{mM} \mathrm{MgSO}_{4}, 25 \mathrm{mM}$ HEPES, $2 \mathrm{mM}$ pyruvate, $2 \% \mathrm{BSA}$ ) for $30 \mathrm{~min}$, IPA3 or vehicle added for $40 \mathrm{~min}$, and then stimulated with insulin for $20 \mathrm{~min}$. Glucose uptake was initiated by the addition of 2-deoxy[1,2,- $\left.{ }^{3} \mathrm{H}\right]$ glucose (Perkin Elmer, Waltham, MA) and uptake terminated after $5 \mathrm{~min}$ by four quick washes with ice cold PBS followed by addition of 250 $\mu \mathrm{l}$ of $1 \mathrm{~N} \mathrm{NaOH}$ for quantitation of $\left[{ }^{3} \mathrm{H}\right]$ using liquid scintillation. Data were normalized for variability in protein concentration, as determined by Bradford assay.

\subsection{Immunoblotting}

Proteins in cell lysates were resolved using 10-12\% SDS-PAGE and transferred to PVDF membranes for immunoblotting. Immunoreactive bands were visualized using ECL, ECL Prime, or Supersignal Femto reagents and imaged using a BioRad Chemi-Doc gel documentation system. Phosphorylated and total ERK1/2 proteins were visualized using goat anti-mouse 680 and anti-rabbit 800 simultaneously for LiCor imaging.

\subsection{Statistical analysis}

All data were expressed as the average \pm SE using Student's $t$-test. Time course and dose response data were evaluated with one-way ANOVA and a Tukey post hoc test using GraphPad Prism $^{\text {TM }}$ (La Jolla, CA).

\section{Results}

\subsection{PAK expression and inhibition by IPA3 in skeletal muscle cells}

To determine if the defects in insulin-stimulated GLUT4 accumulation at the cell surface in the PAK1 KO mice were related to PAK1 signaling, we employed IPA3, an allosteric inhibitor of PAK kinase activation and signaling activity that is selectively initiated by the 
small GTPases Rac1 and Cdc42 [32,33]. IPA3 binds covalently to the PAK1 regulatory domain and prevents binding to these upstream activators, although pre-activated PAK is not inhibited by IPA3. IPA3 is capable of inhibiting all PAK isoforms of the Group 1 family (includes PAKs 1-3), therefore the expression of all three of these isoforms in skeletal muscle was evaluated using immunoblotting and quantitative real-time PCR (qRT-PCR). Of the three existing isoforms, only PAK1 and PAK2 were found in skeletal muscle of C57BL6 mice (Fig. 1A and B), and this pattern was recapitulated by L6-GLUT4-myc myoblasts. PAK1 and PAK2 migrate at distinct molecular weights on SDS-PAGE (68 and $61 \mathrm{kDa}$, respectively), and immunoblotting using a phospho-specific PAK1/2 ${ }^{\mathrm{T} 423 / 402}$ antibody showed that PAK1 displays substantial insulin-stimulated phosphorylation/activation (versus basal $=1.0$, insulin-stimulated $=2.0 \pm 0.3, p<0.05$ ) within 10 min (Fig. 1C). In fewer than half of all experiments using this antibody, PAK2 showed low levels of phosphorylation. By contrast, PAK1 consistently displayed a high level of activation in response to insulin, further confirmed using a PAK $1^{\mathrm{T} 423}$ selective phospho-specific antibody in Fig. 1D (versus basal $=1.0$, insulin-stimulated $=1.6 \pm 0.1, p<0.05$ ).

We next optimized the dosage and time-dependence of IPA3 action in myoblasts, based upon prior experimentation using IPA3 [16]. IPA3 provoked a $>60 \%$ inhibition of insulinstimulated PAK1 phosphorylation at 25 and $30 \mu \mathrm{M}$ (Fig. 2A). At $25 \mu \mathrm{M}$, inhibition of PAK1 activation was consistently achieved within $60 \mathrm{~min}$ (Fig. 2B). No PAK2 phosphorylation was detected in any of these experiments. IPA3 treatment at the most effective time and dose was without impact upon insulin-dependent Akt phosphorylation (Fig. 2C), reminiscent to the lack of impact on Akt phosphorylation displayed by skeletal muscle of PAK1 KO mice [16]. Therefore, $25 \mu \mathrm{M}$ IPA3 treatment for $60 \mathrm{~min}$ was used in subsequent experiments.

\subsection{Insulin-stimulated PAK1 phosphorylation is essential for GLUT4 vesicle translocation and glucose uptake into skeletal muscle cells}

We next examined the effect of IPA3 on insulin-stimulated GLUT4 vesicle exocytosis and glucose uptake. Insulin stimulated a 170\% increase in GLUT4 at the cell surface in myoblasts treated with vehicle alone (DMSO), and IPA3 treatment abrogated this effect of insulin (Fig. 3A). IPA3 did not exert a negative impact upon basal/unstimulated levels of GLUT4 (versus vehicle control $=1.0$, IPA3 $=1.1 \pm 0.1, p>0.05$ ). Glucose uptake was measured in myotubes, since at this stage the contribution of the housekeeping glucose transporter GLUT1 to glucose uptake is minimal. Consistent with the reduction in GLUT4 translocation, IPA3 treatment markedly reduced insulin-stimulated glucose uptake into L6 myotubes (Fig. 3B). In another approach, myoblasts were transfected to express the PAK1 auto-inhibitory domain (PID), a known dominant-negative for PAK1 signaling actions [34,35]. Indeed, PID-expressing myoblasts showed attenuated insulin-stimulated GLUT4 translocation to the cell surface, relative to that of cells expressing the non-inhibitory mutant PID-L107F (Fig. 3C). PID had no negative impact upon basal levels of surface GLUT4 (versus $\mathrm{L} 107 \mathrm{~F}$ control $=1.0, \mathrm{PID}=1.2 \pm 0.1, p>0.05$ ). Since IPA3 also has the capacity to inhibit PAK2, we tested the effect of PAK2 depletion in L6 myoblasts. Transfection with siPAK2 oligonucleotides achieved 75\% PAK2 knockdown relative to non-targeting control oligonucleotides (Fig. 3D), yet this was without effect upon insulin-stimulated GLUT4 
translocation (Fig. 3E). Combined with evidence showing skeletal muscle PAK1 KO to exhibit attenuated insulin-stimulated GLUT4 translocation [16], these data suggest that PAK1 but not PAK2 signaling is required for insulin-stimulated GLUT4 vesicle exocytosis in L6 myoblasts, revealing a selective importance for PAK1 in this process.

\subsection{PAK1 signaling is required for insulin-induced F-actin remodeling in skeletal myoblasts}

Since PAK1 inhibition by IPA3 did not affect Akt phosphorylation, we tested the possibility that PAK1 contributes to the insulin-signaling arm involving PI3K-Rac1 and actin remodeling. Actin remodeling is a prerequisite for GLUT4 vesicle translocation and glucose uptake into L6 myoblasts [36] and mature skeletal muscle [37]. PAK1 is known to impact actin remodeling in other cell types [19,38], either by inducing F-actin formation/assembly or reducing F-actin in a cell type-specific manner. All studies to date have used fixed-cell imaging to study this process, with the inherent caveat that basal and insulin-stimulated images derive from different cells. Instead, we sought to capture the real-time changes in actin polymerization in single cells using live-cell imaging of L6 myoblasts harboring the LifeAct-GFP biosensor. LifeAct is a 17 residue peptide from the actin binding protein Abp140 linked to the N-terminus of GFP to form LifeAct-GFP, which has been shown to bind specifically to F-actin in live cells without adversely affecting F-actin dynamics [25,39]. L6 myoblasts transfected to express LifeAct-GFP exhibited F-actin remodeling within minutes of insulin stimulation, as can be viewed in real-time movies (Supplemental movies 1-4), with still images captured at the end of the $10 \mathrm{~min}$ period (Fig. 4); remodeling is visualized as membrane ruffling (see regions denoted by arrows). Notably, IPA3 treatment completely blocked all insulin-induced F-actin remodeling across the entire imaging period in all independent movies captured. These experiments are the first to demonstrate the time-lapse events of insulin-stimulated actin remodeling in skeletal muscle cells, wherein PAK1 plays an essential role in the process.

\subsection{Insulin-stimulated PAK1 signaling in L6 myoblasts}

We next questioned whether PAK1 signaling to evoke F-actin remodeling occurred via a canonical or non-canonical route. The canonical pathway from PAK1 to evoke cofilin phosphorylation is through activation of LIM kinase (LIMK1/2) in other cell types [40-42]. Incongruent with this, LIMK knockdown in L6 myoblasts failed to substantially affect cofilin phosphorylation [14]. Hence, IPA3 was used to evaluate this canonical pathway, serving as an alternative to depleting PAK1 which otherwise also impedes its scaffolding function. Insulin stimulation triggered a $>2$-fold increase in p-PAK1 activation, and this was inhibited by IPA3 (Fig. 5A); IPA3 was without impact upon basal activation (versus vehicle $=1.0$, IPA3 $=1.0 \pm 0.2, p>0.05$ ), as seen in Fig. 2B. In the same cell lysates, insulin stimulated LIMK phosphorylation by $\sim 2$-fold; however, IPA3 failed to inhibit this activation (Fig. 5B). IPA3-treated lysates showed slightly higher pLIMK levels (versus basal vehicle of $0.5 \pm 0.03$, IPA $3=1.0 \pm 0.07$ ), although since insulin-stimulated pLIMK were similarly altered, the fold activation level was comparable to that of vehicle-treated cells. Issues with pLIMK and LIMK antibodies required that dual gels be used, one for LIMK and the other for pLIMK immunoblotting, with each normalized for deviations in protein loading to actin, and then the pLIMK/total LIMK calculated. The pLIMK antibody recognized multiple non- 
specific bands, and the true pLIMK bands were discerned by paired migration against the correct band in stimulated HUVEC cells as recommended by the manufacturer. As shown previously [14], and opposite to the canonical pathway, insulin stimulation decreased pcofilin levels to $\sim 50 \%$ of that from unstimulated cells. Importantly, IPA3-treated myoblasts showed blunted insulin-stimulated cofilin dephosphorylation (Fig. 5C). Consistent with its lack of effect upon surface GLUT4 or glucose uptake, IPA3 was without effect upon basal levels of cofilin phosphorylation (versus control $=1.0$, IPA3 $=0.9 \pm 0.1, p>0.05$ ). Insulininduced phosphorylation of ERK served as an unrelated pathway control for the PAKspecific effects of IPA3, and to demonstrate that the cells were appropriately responsive to insulin (Fig. 5D). Taken together, these findings highlight the importance of PAK1 signaling for cofilin dephosphorylation, which proceeds through a non-canonical, LIMK-independent pathway.

\section{Discussion}

PAK1 KO mice exhibit peripheral insulin resistance [16]. However, since PAK1 is known to function both as a scaffolding protein and as a signaling protein [23], its ablation in the PAK1 KO mouse model left elucidation of its specific function(s) in regulating insulin sensitivity unresolved. Moreover, this whole body knockout model of PAK1 precluded assigning its mode of action directly to skeletal muscle. Here, we show that insulinstimulated PAK1 activation is an integral step in the signaling cascade that enables actin remodeling and GLUT4 vesicle translocation to stimulate glucose uptake into skeletal muscle cells. Showing for the first time the kinetics of insulin-stimulated actin remodeling in live skeletal muscle cells by time-lapse imaging using the F-actin biosensor LifeAct-GFP, we reveal that PAK1 activation is required to display this actin remodeling. Since IPA3mediated PAK1 inhibition failed to impair Akt phosphorylation but did impair actin remodeling, PAK1 signaling is likely required in the Akt-independent PI3K $\rightarrow$ Rac1 signaling cascade of insulin action. Prior work shows that insulin-stimulated cofilin dephosphorylation is required for GLUT4 translocation [14]; our data fully recapitulate this work. Mechanistically, our data expand upon this to show that IPA3-mediated inhibition of p-PAK1 abrogates the dephosphorylation of cofilin, a finding incongruent with the canonical, stimulus-induced p-PAK1 $\rightarrow$ p-LIMK $\rightarrow$ p-cofilin response. Moreover, cofilin dephosphorylation occurred in a manner independent of LIMK, suggesting that PAK1 contributes to actin remodeling via a non-canonical pathway.

The requirement for PAK1 signaling to evoke the dephosphorylation of cofilin is unusual, only otherwise reported to occur in the MCF-7 cells [43]. PAK1 signals to numerous substrates in a variety of cell types [44], one of which is LIMK [42], which in turn leads to the phosphorylation (inactivation) of cofilin to regulate the actin cytoskeletal dynamics. In contrast, insulin-stimulated actin remodeling in skeletal muscle cells involves cofilin dephosphorylation [14]. The model drawn from these findings proposes that GLUT4 vesicle translocation and fusion requires active cycling or 'remodeling' of actin. Our live-cell imaging demonstrates this dynamic cycling to occur in response to insulin, and that PAK1 signaling is required to facilitate the cycling. Supporting this concept in the context of cofilin dephosphorylation by insulin, Slingshot1 (SSH1) was identified as the cofilin phosphatase in the L6 muscle cells [14]. SSH1 activity was shown to predominate over that 
of LIMK in these muscle cells, with LIMK knockdown having little impact upon cofilin phosphorylation status. Our data support this and further show that while PAK1 is upstream of cofilin, IPA3-mediated inhibition of PAK1 fails to inhibit LIMK activation. Alternative to activation by PAK1, LIMK may become activated in response to insulin stimulation via Rho kinase or 'ROCK' activation [45]. Regardless though, this LIMK pathway is expected to be a lesser contributor to actin remodeling given the substantial impact of IPA3-mediated PAK1 inhibition. An additional open question is whether SSH1 activity is regulated by PAK1, either directly or indirectly. SSH1 is activated upon its dephosphorylation, in response to calcineurin [46] as well as to formation of polymerized actin; SSH1 contains an actin binding domain [47]. SSH1 is inactivated by protein kinase D1, phosphorylating a serine residue located in its actin-binding motif [48]. The PAK4 isoform, a Group II PAK, is known to negatively regulate SSH1 activity [49], although this is the opposite of what one would expect given that in skeletal muscle cells, cofilin is dephosphorylated in response to insulin and in a pPAK1-dependent manner. Hence, our data would appear to be consistent with PAK1 acting upstream as an indirect regulator of SSH1, via its ability to induce actin polymerization.

In L6 skeletal muscle cells, cofilin dephosphorylation requires a prior accumulation of polymerized actin driven by Arp2/3 [14]. The Arp2/3 complex, an actin-nucleating complex in association with cofilin, is implicated in the regulation of cortical actin remodeling in skeletal muscle cells. In other cell types PAK1 is known to regulate actin remodeling by phosphorylating p41ARC, a regulatory subunit of Arp2/3 complex [50]. Additionally, the Arp2/3 complex with its activator N-WASP is involved in the recycling of GLUT4 transporters via regulating cortical actin remodeling in adipocytes [51]. Studies are underway to investigate the role and placement of p41ARC and N-WASP in the PAK1dependent insulin signaling pathway in skeletal muscle cells. Additionally, PAK1 signaling to other downstream factors such as cortactin, Myo1c and Filamin A should be investigated, since these targets are known to bind to PAK1 in non-muscle cells and have been implicated as indirect effectors of glucose uptake [52-56].

In L6-GLUT4-myc myotubes the depletion of Rac1 results in the ablation of PAK1 activation [10], as does the treatment of mouse skeletal muscle with the Rac1 inhibitor NSC23766 [13]. Moreover, skeletal muscle-specific Rac1 KO mice have impaired skeletal muscle PAK1 signaling, suggesting that PAK1 is downstream of Rac1 [13]. However, PAK1 has also been implied to function upstream of Rac1 in other cell types, by phosphorylating RhoGDI to yield activated Rac1 [57]. Hence, future studies are required to investigate the potential additional placement of PAK1 upstream of Rac1 using PAK1 inhibitors and skeletal muscle-specific PAK1 KO mice.

The fact that IPA3 action on GLUT4 translocation and cofilin phosphorylation status is reminiscent to that of PAK1 depletion in vivo suggests that its actions on muscle cells are unlikely to arise from effects on targets other than PAK1. However, we cannot discount that the inhibitory action on actin remodeling is entirely due to its action on PAK1.

In summary, we have demonstrated that PAK1 signals to cofilin in response to insulin and facilitates the cortical actin remodeling in skeletal muscle cells. With this, PAK1 mediates 
the GLUT4 vesicle translocation to the cell surface and results in glucose uptake into the skeletal muscle cells. Interestingly, a GWAS study has localized PAK1 to the T2D susceptibility locus on human Chromosome 11 [58,59], and PAK1 deficiency is linked to glucose intolerance, insulin resistance and T2D in human and rodent models of these pathophysiological states $[13,16,60]$. Altogether, these data suggest that a decrease in PAK1 abundance/function might be a potential risk factor for pre-diabetes susceptibility. Hence strategies/methods to restore the function or abundance of PAK1 in skeletal muscle could be useful for re-establishing insulin action and euglycemia.

\section{Supplementary Material}

Refer to Web version on PubMed Central for supplementary material.

\section{Acknowledgments}

We would like to thank Lixuan Tackett and Dr. Brent Penque (Indiana University School of Medicine, Indianapolis, IN) for technical assistance with the L6-GLUT4-myc cell experiments, as well as Dr. Mike Kalwat (Indiana University School of Medicine, Indianapolis, IN) and the O'Brien Microscopy core facility at Indiana University School of Medicine regarding the live-cell F-actin remodeling imaging. We also thank Drs. Joe Brozinick, Susan Gunst, Simon Atkinson, Zhanxiang Wang and Stephanie Yoder for critical review of this project. This study was supported by grants from the National Institutes of Health (DK067912 and DK076614 to D.C.T.), and by grant MT3707 from the Canadian Institutes of Health Research to A.K.

\section{References}

1. Leto D, Saltiel AR. Regulation of glucose transport by insulin: traffic control of GLUT4. Nat Rev Mol Cell Biol. 2012; 13:383-96. [PubMed: 22617471]

2. Foley K, Boguslavsky S, Klip A. Endocytosis, recycling, and regulated exocytosis of glucose transporter 4. Biochemistry. 2011; 50:3048-61. [PubMed: 21405107]

3. Bogan JS, Kandror KV. Biogenesis and regulation of insulin-responsive vesicles containing GLUT4. Curr Opin Cell Biol. 2010; 22:506-12. [PubMed: 20417083]

4. DeFronzo RA, Tripathy D. Skeletal muscle insulin resistance is the primary defect in type 2 diabetes. Diabetes Care. 2009; 32(Suppl 2):S157-63. [PubMed: 19875544]

5. Karlsson HK, Zierath JR. Insulin signaling and glucose transport in insulin resistant human skeletal muscle. Cell Biochem Biophys. 2007; 48:103-13. [PubMed: 17709880]

6. DeFronzo RA, Gunnarsson R, Bjorkman O, Olsson M, Wahren J. Effects of insulin on peripheral and splanchnic glucose metabolism in noninsulin-dependent (Type II) diabetes mellitus. J Clin Invest. 1985; 76:149-55. [PubMed: 3894418]

7. Bruss MD, Arias EB, Lienhard GE, Cartee GD. Increased phosphorylation of Akt substrate of 160 $\mathrm{kDa}(\mathrm{AS} 160)$ in rat skeletal muscle in response to insulin or contractile activity. Diabetes. 2005; 54:41-50. [PubMed: 15616009]

8. Klip A, Sun Y, Chiu TT, Foley KP. Signal transduction meets vesicle traffic: the software and hardware of GLUT4 translocation. Am J Physiol Cell Physiol. 2014; 306:C879-86. [PubMed: 24598362]

9. Bosco EE, Mulloy JC, Zheng Y. Rac1 GTPase: a "Rac" of all trades. Cell Mol Life Sci. 2009; 66:370-4. [PubMed: 19151919]

10. JeBailey L, Wanono O, Niu W, Roessler J, Rudich A, Klip A. Ceramide- and oxidant-induced insulin resistance involve loss of insulin-dependent Rac-activation and actin remodeling in muscle cells. Diabetes. 2007; 56:394-403. [PubMed: 17259384]

11. Ueda S, Kitazawa S, Ishida K, Nishikawa Y, Matsui M, Matsumoto H, et al. Crucial role of the small GTPase Rac1 in insulin-stimulated translocation of glucose transporter 4 to the mouse skeletal muscle sarcolemma. FASEB J. 2010; 24:2254-61. [PubMed: 20203090] 
12. Chiu TT, Jensen TE, Sylow L, Richter EA, Klip A. Rac1 signalling towards GLUT4/glucose uptake in skeletal muscle. Cell Signal. 2011; 23:1546-54. [PubMed: 21683139]

13. Sylow L, Jensen TE, Kleinert M, Hojlund K, Kiens B, Wojtaszewski J, et al. Rac1 signaling is required for insulin-stimulated glucose uptake and is dysregulated in insulin-resistant murine and human skeletal muscle. Diabetes. 2013; 62:1865-75. [PubMed: 23423567]

14. Chiu TT, Patel N, Shaw AE, Bamburg JR, Klip A. Arp2/3- and cofilin-coordinated actin dynamics is required for insulin-mediated GLUT4 translocation to the surface of muscle cells. Mol Biol Cell. 2010; 21:3529-39. [PubMed: 20739464]

15. Tsakiridis T, Taha C, Grinstein S, Klip A. Insulin activates a p21-activated kinase in muscle cells via phosphatidylinositol 3-kinase. J Biol Chem. 1996; 271:19664-67. [PubMed: 8702668]

16. Wang Z, Oh E, Clapp DW, Chernoff J, Thurmond DC. Inhibition or ablation of p21-activated kinase (PAK1) disrupts glucose homeostatic mechanisms in vivo. J Biol Chem. 2011; 286:41359_ 67. [PubMed: 21969371]

17. Chiang YA, Shao W, Xu XX, Chernoff J, Jin T. P21-activated protein kinase 1 (Pak1) mediates the cross talk between insulin and beta-catenin on proglucagon gene expression and its ablation affects glucose homeostasis in male C57BL/6 mice. Endocrinology. 2013; 154:77-88. [PubMed: 23183186]

18. Wang Z, Oh E, Thurmond DC. Glucose-stimulated Cdc42 signaling is essential for the second phase of insulin secretion. J Biol Chem. 2007; 282:9536-46. [PubMed: 17289663]

19. Kalwat MA, Yoder SM, Wang Z, Thurmond DC. A p21-activated kinase (PAK1) signaling cascade coordinately regulates F-actin remodeling and insulin granule exocytosis in pancreatic beta cells. Biochem Pharmacol. 2013; 85:808-16. [PubMed: 23246867]

20. Chen YC, Fueger PT, Wang Z. Depletion of PAK1 enhances ubiquitin-mediated Survivin degradation in pancreatic beta-cells. Islets. 2013; 5:22-8. [PubMed: 23514967]

21. Liu W, Zi M, Naumann R, Ulm S, Jin J, Taglieri DM, et al. Pak1 as a novel therapeutic target for antihypertrophic treatment in the heart. Circulation. 2011; 124:2702-15. [PubMed: 22082674]

22. Lim GE, Huang GJ, Flora N, LeRoith D, Rhodes CJ, Brubaker PL. Insulin regulates glucagon-like peptide-1 secretion from the enteroendocrine L cell. Endocrinology. 2009; 150:580-91. [PubMed: 18818290]

23. Frost JA, Khokhlatchev A, Stippec S, White MA, Cobb MH. Differential effects of PAK1activating mutations reveal activity-dependent and -independent effects on cytoskeletal regulation. J Biol Chem. 1998; 273:28191-98. [PubMed: 9774440]

24. Walker PS, Ramlal T, Sarabia V, Koivisto UM, Bilan PJ, Pessin JE, et al. Glucose transport activity in L6 muscle cells is regulated by the coordinate control of subcellular glucose transporter distribution, biosynthesis, and mRNA transcription. J Biol Chem. 1990; 265:1516-23. [PubMed: 2404015]

25. Lopez JP, Turner JR, Philipson LH. Glucose-induced ERM protein activation and translocation regulates insulin secretion. Am J Physiol Endocrinol Metab. 2010; 299:E772-85. [PubMed: 20739507]

26. Arias-Romero LE, Chernoff J. p21-activated kinases in Erbb2-positive breast cancer: a new therapeutic target? Small GTPases. 2010; 1:124-8. [PubMed: 21686266]

27. Kanai F, Nishioka Y, Hayashi H, Kamohara S, Todaka M, Ebina Y. Direct demonstration of insulin-induced GLUT4 translocation to the surface of intact cells by insertion of a c-myc epitope into an exofacial GLUT4 domain. J Biol Chem. 1993; 268:14523-26. [PubMed: 7686158]

28. Ueyama A, Yaworsky KL, Wang Q, Ebina Y, Klip A. GLUT-4myc ectopic expression in L6 myoblasts generates a GLUT-4-specific pool conferring insulin sensitivity. Am J Physiol. 1999; 277:E572-8. [PubMed: 10484371]

29. Foley KP, Klip A. Dynamic GLUT4 sorting through a syntaxin-6 compartment in muscle cells is derailed by insulin resistance-causing ceramide. Biol Open. 2014; 3:314-25. [PubMed: 24705014]

30. Habegger KM, Penque BA, Sealls W, Tackett L, Bell LN, Blue EK, et al. Fat-induced membrane cholesterol accrual provokes cortical filamentous actin destabilisation and glucose transport dysfunction in skeletal muscle. Diabetologia. 2012; 55:457-67. [PubMed: 22002007] 
31. McCarthy AM, Spisak KO, Brozinick JT, Elmendorf JS. Loss of cortical actin filaments in insulinresistant skeletal muscle cells impairs GLUT4 vesicle trafficking and glucose transport. Am J Physiol Cell Physiol. 2006; 291:C860-8. [PubMed: 16774991]

32. Deacon SW, Beeser A, Fukui JA, Rennefahrt UE, Myers C, Chernoff J, et al. An isoform-selective, small-molecule inhibitor targets the autoregulatory mechanism of p21-activated kinase. Chem Biol. 2008; 15:322-31. [PubMed: 18420139]

33. Viaud J, Peterson JR. An allosteric kinase inhibitor binds the p21-activated kinase autoregulatory domain covalently. Mol Cancer Ther. 2009; 8:2559-65. [PubMed: 19723886]

34. Delorme-Walker VD, Peterson JR, Chernoff J, Waterman CM, Danuser G, DerMardirossian C, et al. Pak1 regulates focal adhesion strength, myosin IIA distribution, and actin dynamics to optimize cell migration. J Cell Biol. 2011; 193:1289-303. [PubMed: 21708980]

35. Zenke FT, King CC, Bohl BP, Bokoch GM. Identification of a central phosphorylation site in p21activated kinase regulating autoinhibition and kinase activity. J Biol Chem. 1999; 274:32565-73. [PubMed: 10551809]

36. Tsakiridis T, Vranic M, Klip A. Disassembly of the actin network inhibits insulin-dependent stimulation of glucose transport and prevents recruitment of glucose transporters to the plasma membrane. J Biol Chem. 1994; 269:29934-42. [PubMed: 7961991]

37. Brozinick JT Jr, Hawkins ED, Strawbridge AB, Elmendorf JS. Disruption of cortical actin in skeletal muscle demonstrates an essential role of the cytoskeleton in glucose transporter 4 translocation in insulin-sensitive tissues. J Biol Chem. 2004; 279:40699-706. [PubMed: 15247264]

38. Zhu J, Attias O, Aoudjit L, Jiang R, Kawachi H, Takano T. p21-activated kinases regulate actin remodeling in glomerular podocytes. Am J Physiol Renal Physiol. 2010; 298:F951-61. [PubMed: 20071462]

39. Rondas D, Tomas A, Halban PA. Focal adhesion remodeling is crucial for glucose-stimulated insulin secretion and involves activation of focal adhesion kinase and paxillin. Diabetes. 2011; 60:1146-57. [PubMed: 21357465]

40. Gerthoffer WT. Mechanisms of vascular smooth muscle cell migration. Circ Res. 2007; 100:60721. [PubMed: 17363707]

41. Scott RW, Hooper S, Crighton D, Li A, Konig I, Munro J, et al. LIM kinases are required for invasive path generation by tumor and tumor-associated stromal cells. J Cell Biol. 2010; 191:16985. [PubMed: 20876278]

42. Edwards DC, Sanders LC, Bokoch GM, Gill GN. Activation of LIM-kinase by Pak1 couples Rac/ Cdc42 GTPase signalling to actin cytoskeletal dynamics. Nat Cell Biol. 1999; 1:253-9. [PubMed: 10559936]

43. Coniglio SJ, Zavarella S, Symons MH. Pak1 and Pak2 mediate tumor cell invasion through distinct signaling mechanisms. Mol Cell Biol. 2008; 28:4162-72. [PubMed: 18411304]

44. Ong CC, Jubb AM, Zhou W, Haverty PM, Harris AL, Belvin M, et al. p21-activated kinase 1: PAK'ed with potential. Oncotarget. 2011; 2:491-6. [PubMed: 21653999]

45. Maekawa M, Ishizaki T, Boku S, Watanabe N, Fujita A, Iwamatsu A, et al. Signaling from Rho to the actin cytoskeleton through protein kinases ROCK and LIM-kinase. Science. 1999; 285:895-8. [PubMed: 10436159]

46. Zhao JW, Gao ZL, Ji QY, Wang H, Zhang HY, Yang YD, et al. Regulation of cofilin activity by CaMKII and calcineurin. Am J Med Sci. 2012; 344:462-72. [PubMed: 22270398]

47. Kurita S, Gunji E, Ohashi K, Mizuno K. Actin filaments-stabilizing and -bundling activities of cofilin-phosphatase Slingshot-1. Genes Cells. 2007; 12:663-76. [PubMed: 17535256]

48. Eiseler T, Doppler H, Yan IK, Kitatani K, Mizuno K, Storz P. Protein kinase D1 regulates cofilinmediated F-actin reorganization and cell motility through slingshot. Nat Cell Biol. 2009; 11:54556. [PubMed: 19329994]

49. Soosairajah J, Maiti S, Wiggan O, Sarmiere P, Moussi N, Sarcevic B, et al. Interplay between components of a novel LIM kinase-slingshot phosphatase complex regulates cofilin. EMBO J. 2005; 24:473-86. [PubMed: 15660133] 
50. Vadlamudi RK, Li F, Barnes CJ, Bagheri-Yarmand R, Kumar R. p41-Arc subunit of human Arp2/3 complex is a p21-activated kinase-1-interacting substrate. EMBO Rep. 2004; 5:154-60. [PubMed: 14749719]

51. Jiang ZY, Chawla A, Bose A, Way M, Czech MP. A phosphatidylinositol 3-kinase-independent insulin signaling pathway to N-WASP/Arp2/3/F-actin required for GLUT4 glucose transporter recycling. J Biol Chem. 2002; 277:509-515. [PubMed: 11694514]

52. Nazari H, Khaleghian A, Takahashi A, Harada N, Webster NJ, Nakano M, et al. Cortactin, an actin binding protein, regulates GLUT4 translocation via actin filament remodeling. Biochemistry (Mosc). 2011; 76:1262-9. [PubMed: 22117553]

53. Webb BA, Zhou S, Eves R, Shen L, Jia L, Mak AS. Phosphorylation of cortactin by p21-activated kinase. Arch Biochem Biophys. 2006; 456:183-93. [PubMed: 16854367]

54. Bose A, Robida S, Furcinitti PS, Chawla A, Fogarty K, Corvera S, et al. Unconventional myosin Myo1c promotes membrane fusion in a regulated exocytic pathway. Mol Cell Biol. 2004; 24:5447-58. [PubMed: 15169906]

55. Bose A, Guilherme A, Robida SI, Nicoloro SM, Zhou QL, Jiang ZY, et al. Glucose transporter recycling in response to insulin is facilitated by myosin Myo1c. Nature. 2002; 420:821-4. [PubMed: 12490950]

56. Deshmukh A, Coffey VG, Zhong Z, Chibalin AV, Hawley JA, Zierath JR. Exercise-induced phosphorylation of the novel Akt substrates AS160 and filamin A in human skeletal muscle. Diabetes. 2006; 55:1776-82. [PubMed: 16731842]

57. DerMardirossian C, Schnelzer A, Bokoch GM. Phosphorylation of RhoGDI by Pak1 mediates dissociation of Rac GTPase. Mol Cell. 2004; 15:117-27. [PubMed: 15225553]

58. Silander K, Scott LJ, Valle TT, Mohlke KL, Stringham HM, Wiles KR, et al. A large set of Finnish affected sibling pair families with type 2 diabetes suggests susceptibility loci on chromosomes 6 , 11, and 14. Diabetes. 2004; 53:821-9. [PubMed: 14988269]

59. Palmer ND, Langefeld CD, Campbell JK, Williams AH, Saad M, Norris JM, et al. Genetic mapping of disposition index and acute insulin response loci on chromosome 11q. The Insulin Resistance Atherosclerosis Study (IRAS) Family Study. Diabetes. 2006; 55:911-8. [PubMed: 16567510]

60. Keller MP, Choi Y, Wang P, Davis DB, Rabaglia ME, Oler AT, et al. A gene expression network model of type 2 diabetes links cell cycle regulation in islets with diabetes susceptibility. Genome Res. 2008; 18:706-16. [PubMed: 18347327]

\section{Appendix A. Supplementary data}

Supplementary data associated with this article can be found, in the online version, at http:// dx.doi.org/10.1016/j.bcp.2014.08.033. 
A
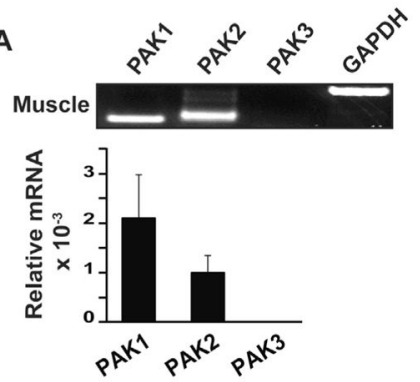

C

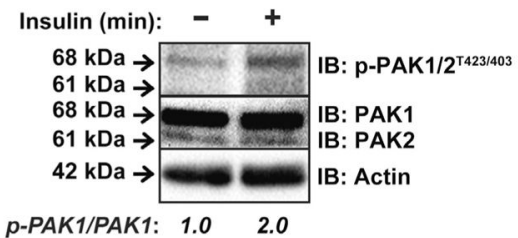

B

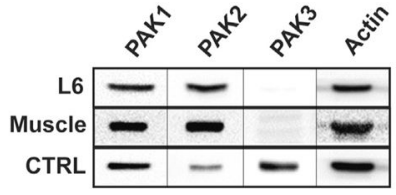

D

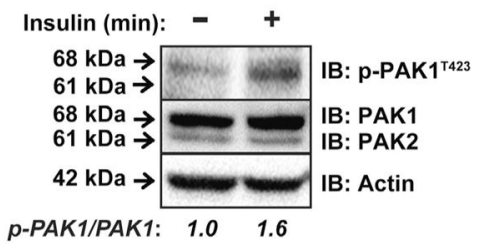

Fig. 1.

Group I PAK isoforms in skeletal muscle and L6-GLUT4-myc cells. Hindlimb muscle from wild-type (WT) mice was homogenized and analyzed for the three Group I PAK members PAK1, 2 and 3 for (A) mRNA content using qRT-PCR (normalized to GAPDH) from three sets of tissues, and (B) protein expression. L6-GLUT4-myc myoblasts were assessed similarly for protein expression of all three Group I PAKs; mouse brain lysates served as control (CTRL) as they co-express all three isoforms. Vertical black lines denote splicing of lanes from the PAK1, PAK2, PAK3 and actin immunoblots. (C) Lysate proteins from L6GLUT4-myc myoblast cells left unstimulated or insulin-stimulated (100 nM, $10 \mathrm{~min}$ ) were resolved by $12 \%$ SDS-PAGE for immunoblot detection of phosphorylated- and total- PAK1 and PAK2 proteins and (D) specifically for phosphorylated PAK1. Data are representative of three independent experiments, with average insulin-stimulated p-PAK1/total PAK1 ratios shown below the blots, normalized to basal $=1.0$ for each experiment. 
A

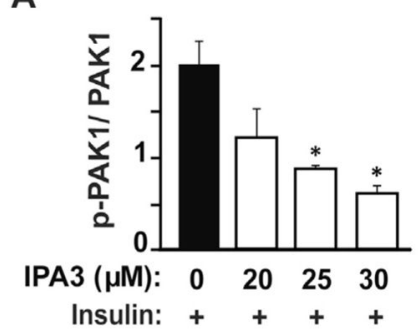

B
IPA3 (min): $0 \quad 20 \quad 30 \quad 60$
Insulin: $-+\frac{20}{-++}+\frac{30}{-+}$

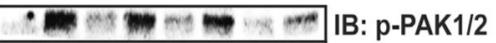
$=-m=-m$ IB: PAK1
$-\infty=-\infty$ IB: Actin

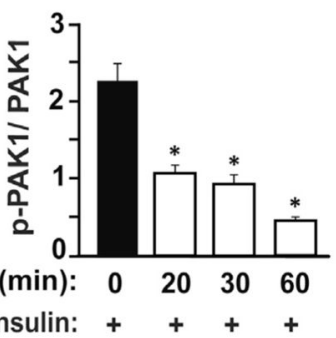

C

IPA3 (min): - - + +

Insulin: - + - +

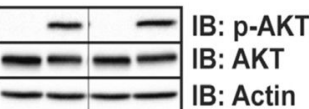

IB: Actin

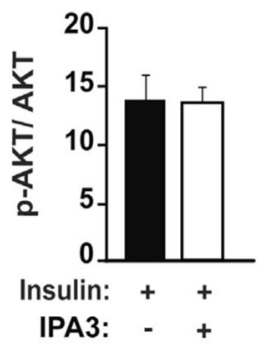

Fig. 2.

IPA3 inhibits insulin-stimulated PAK1 phosphorylation in L6-GLUT4-myc myoblasts. (A) Dose-optimization: L6 myoblasts were pretreated with IPA3 at 20, 25 or $30 \mu \mathrm{M}$ for $10 \mathrm{~min}$ followed by insulin stimulation for an additional $10 \mathrm{~min}$. Detergent cleared cell lysates were prepared and proteins resolved by SDS-PAGE for immunoblot and optical density scanning quantitation for p-PAK1 relative to total PAK1 (in arbitrary units). (B) Time course: L6 myoblasts were treated with $25 \mu \mathrm{M}$ IPA3 for $0,20,40$ or $60 \mathrm{~min}$, with insulin added in the final $10 \mathrm{~min}(100 \mathrm{nM})$. Cell lysates were immunoblotted for p-PAK1 and PAK1 and 
quantified as in (A) above. (C) p-AKT and total AKT were immunoblotted and quantified from cells treated with vehicle (-) or $25 \mu \mathrm{M}$ IPA3 for $60 \mathrm{~min}$ as described in (A) above. Bar graphs represent the average $\pm \mathrm{SE}$ of three independent cell passages; ${ }^{*} p<0.05$. The vertical black line denotes splicing of lanes from within the same gel exposure. 
A

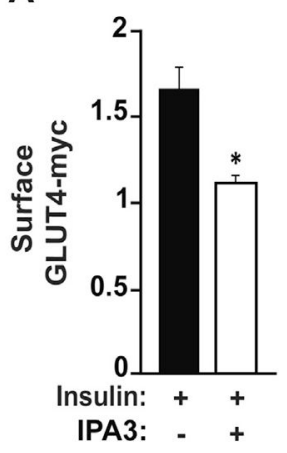

B

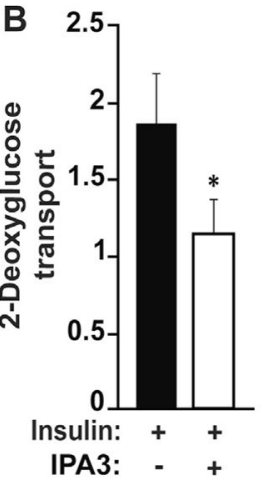

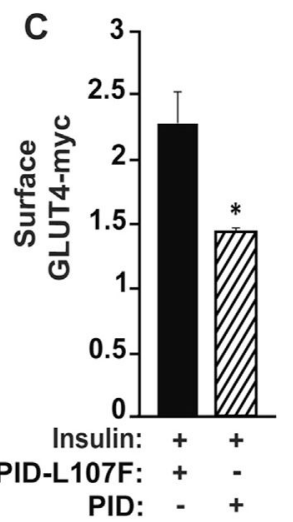
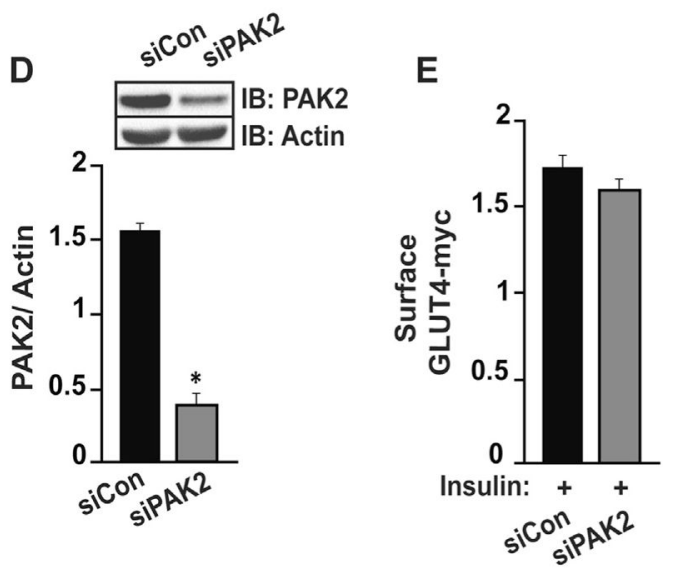

Fig. 3.

PAK1 activity is essential for insulin stimulated GLUT4 vesicle translocation and glucose uptake in skeletal muscle cells. (A) Surface GLUT4: L6-GLUT4-myc myoblasts treated with IPA3 or vehicle (DMSO) were stimulated with insulin (100 nM, $20 \mathrm{~min}$ ) for LiCor analyses of surface GLUT4 levels. Immunofluorescent intensity of cell surface GLUT4 was normalized to nucleic acid staining dye, Syto 60, and data displayed as the fold-stimulation of insulin-stimulated surface GLUT4, relative to unstimulated/basal level. Bars represent the average \pm SE of four independent cell passages; ${ }^{*} p<0.05$, vs vehicle (-) treated cells. (B) Glucose uptake: L6-GLUT4-myc myotubes were used for 2-deoxyglucose uptake assays as described in Section 2. Data represent the average $( \pm \mathrm{SE})$ fold stimulation in response to insulin relative to basal level glucose uptake, in at least three independent passages of cells; ${ }^{*} p<0.05$, vs vehicle (-) treated cells. (C) L6-GLUT4-myc myoblasts were transfected to express GFP-tagged PAK1 inhibitory domain (PID) or non-inhibitory control (PID-L107F) and assessed for insulin-stimulated GLUT4 as described in (A) above. Bars represent the average \pm SE of three independent cell passages; ${ }^{*} p<0.05$, vs PID-L107F-expressing cells. (D) Myoblasts were transfected with control (siCon) or PAK2-selective (siPAK2) siRNA oligonucleotides, normalized to actin (arbitrary units). Bars represent the average \pm SE of three independent cell passages; * $p<0.05$, vs siCon. (E) GLUT4 vesicle translocation 
assays from siPAK2 or control (siCon) transfected cells, as described in (A) above. No significant differences were detected in four independent experiments. 
Insulin:
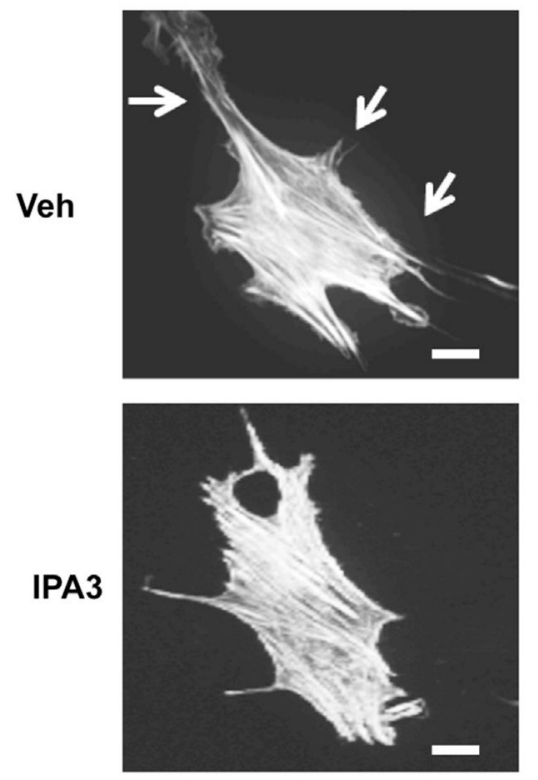

$+$
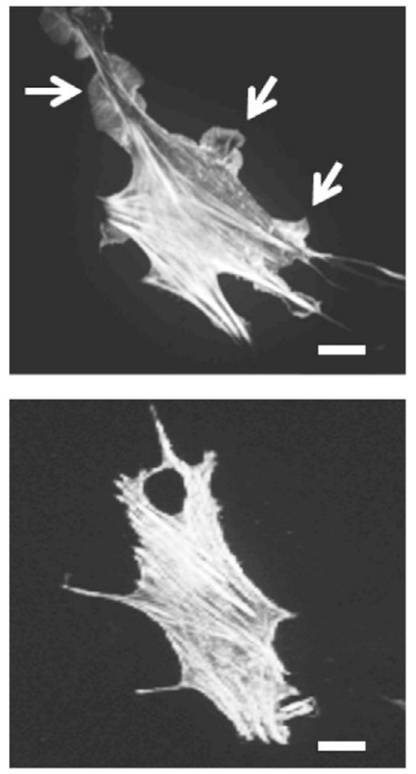

Fig. 4.

Insulin-induced PAK1 signaling is important for insulin-induced F-actin remodeling in L6GLUT4-myc myoblasts. L6-GLUT4-myc myoblasts were transfected to express the LifeAct-GFP biosensor for live-cell imaging of F-actin remodeling. Myoblasts treated with vehicle or IPA3 were imaged using an custom spinning-disk confocal microscope and captured every min from $1 \mathrm{~min}$ prior insulin addition and on through to $10 \mathrm{~min}$ after insulin stimulation $(100 \mathrm{nM})$. Arrows denote regions of ruffling. Images represent still images taken from at least four cell movies of each treatment condition conducted using four independent passages of cells. Movies are included as Supplemental movies 1-4, two movies for each condition shown here. 
A
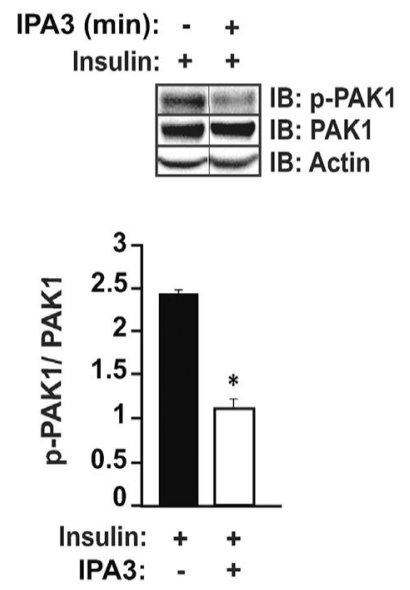

C
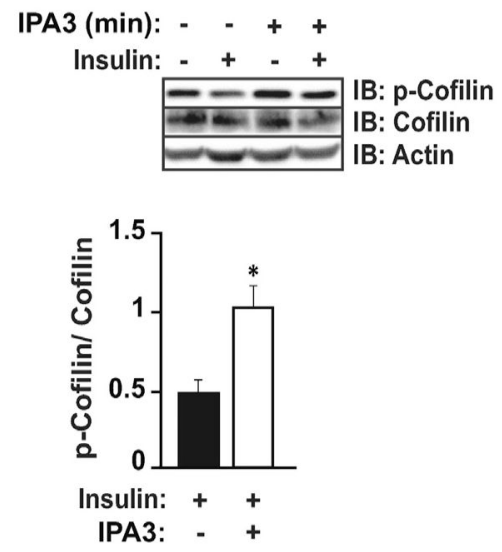

B
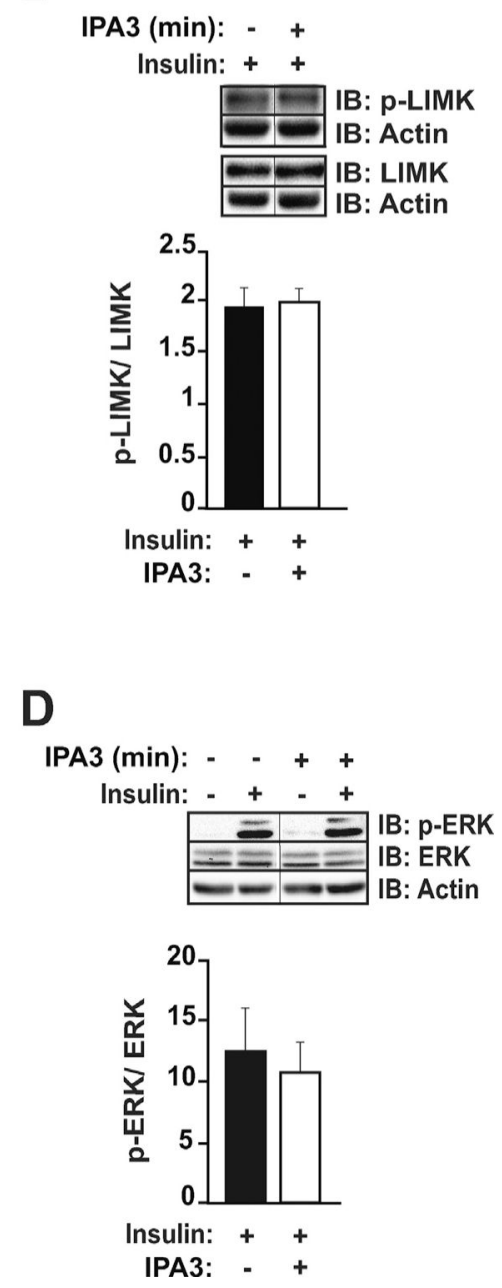

Fig. 5.

PAK1 signaling is required selectively for insulin-stimulated cofilin dephosphorylation in L6-GLUT4-myc myoblasts. L6-GLUT4-myc myoblasts treated with vehicle or IPA3 were stimulated with insulin $(100 \mathrm{nM}, 10 \mathrm{~min})$ and resulting detergent cleared cell lysates resolved on $12 \%$ SDS-PAGE for simultaneous immunoblot detection of (A) p-PAK1 and total PAK1, (B) p-LIMK and total LIMK, (C) p-cofilin and total cofilin, and (D) p-ERK and total ERK. Optical density scanning was used for quantification, with bar graphs representing the average \pm SE of fold changes in activations evaluated in four independent experiments; $* p<0.05$. Vertical black lines denote splicing of lanes from within the same gel exposure within each figure panel. 